OPEN ACCESS

Edited by:

Katja Teerds,

Wageningen University, Netherlands

Reviewed by:

Roger Hart,

University of Western Australia,

Australia

Svetlana Vujovic,

University of Belgrade, Serbia

*Correspondence:

Xiaofeng Yang

yxf73@163.com

Specialty section:

This article was submitted to

Reproduction,

a section of the journal

Frontiers in Endocrinology

Received: 10 February 2020 Accepted: 04 December 2020

Published: 18 January 2021

Citation:

Wang L, LV S, Li F, Bai E and Yang $X$ (2021) Letrozole Versus Clomiphene Citrate and Natural Cycle: Endometrial Receptivity During Implantation Window in Women With

Polycystic Ovary Syndrome.

Front. Endocrinol. 11:532692. doi: 10.3389/fendo.2020.532692

\section{Letrozole Versus Clomiphene Citrate and Natural Cycle: Endometrial Receptivity During Implantation Window in Women With Polycystic Ovary Syndrome}

\author{
Li Wang, Shulan Lv, Fen Li, E. Bai and Xiaofeng Yang * \\ Department of Obstetrics and Gynecology, The First Affiliated Hospital of Xi'an Jiaotong University, Xi'an, China
}

Objective: Numerous studies have reported on ovulation and pregnancy rates in patients with polycystic ovary syndrome (PCOS). However, relevant data on endometrial receptivity are limited. This study was conducted to compare endometrial receptivity during implantation windows among letrozole (LE), clomiphene citrate (CC), and natural cycle, and to assess the predictive value for pregnancy of observed indicators.

Methods: This randomized controlled trial study enrolled 270 patients with PCOS. Patients were given LE $(n=90)$ at a dose of $2.5 \mathrm{mg} /$ day or CC $(n=90)$ at a dose of 50 $\mathrm{mg} /$ day on cycle days 5-9 for ovulation induction. Patients in the natural cycle group $(n=90)$ did not receive any drug for ovulation induction. Endometrial ultrasonic parameters, integrin $\alpha v \beta 3$, and vascular endothelial growth factor (VEGF) concentrations in uterine secretion were detected during the implantation window. The endometrial receptivity, ovulation rate, pregnancy rates, and predictive value of observed indicators for pregnancy were analyzed.

Results: The successful ovulation rate did not differ between the LE group and CC group $(P>0.05)$. Endometrial ultrasonic parameters [endometrial thickness $(E T)$, endometrial volume $(E V)$, vascularization index $(\mathrm{VI})$, flow index $(\mathrm{FI})$, vascularization flow index $(\mathrm{VFI})$ ], integrin $\alpha v \beta 3$, and VEGF concentrations in uterine fluid were significantly higher in the LE group compared with the CC group and natural cycle group $(P<0.05)$. The clinical pregnancy and ongoing pregnancy rates of the LE group were significantly higher than in the CC group $(P<0.05)$. Endometrial ultrasonic parameters $(\mathrm{VI}, \mathrm{FI}$, and $\mathrm{VFI})$, integrin $\alpha v \beta 3$, and VEGF concentrations in uterine fluid of all pregnancy groups were significantly higher compared with the no pregnancy group $(P<0.05)$, and the above parameters in ongoing pregnancy were significantly higher than in biochemical pregnancy $(P<0.05)$. The endometrial $\mathrm{Fl}$ during the implantation window had the highest predictive value for 
pregnancy $(A \cup C=0.889)$. The integrin $\alpha v \beta 3$ in uterine fluid had better predictive value (AUC=0.876) than VEGF.

Conclusions: Endometrial receptivity during the implantation window of LE is superior to CC in PCOS women, which may be related to higher clinical pregnancy and ongoing pregnancy rates. Endometrial FI examined by 3-D power Doppler, and integrin $\alpha \mathrm{v} \beta 3$ in uterine secretion during the implantation window, could be preferable non-invasive predictor markers for pregnancy.

Clinical Trial Registration: www.chictr.org.cn, ChiCTR1900023423.

Keywords: polycystic ovary syndrome, letrozole, clomiphene citrate, endometrial receptivity, embryo implantation

\section{INTRODUCTION}

Polycystic ovary syndrome (PCOS) is an endocrine and metabolic disorder in women of reproductive age, and its prevalence rate is from $9 \%$ (NIH criteria) to $18 \%$ (Rotterdam criteria) (1). The clinical manifestations of PCOS are menstrual irregularities, anovulatory infertility, hyperandrogenism, and disorders of glucose and lipid metabolism $(2,3)$. About $25 \%-$ $30 \%$ of PCOS women of reproductive age need to seek help because of ovulatory dysfunction infertility (4). Clomiphene citrate (CC) promotes follicular development through blocking the negative feedback of estrogen to the hypothalamus and making the pituitary secrete gonadotropin. Therefore, CC has been used as the traditional first-line medication for inducing ovulation in PCOS women, but the antiestrogenic effect of CC on cervical mucus and endometrial receptivity results in low pregnancy rates (5). Letrozole (LE) was initially applied to treat breast cancer through preventing the conversion of androgens to estrogen and reducing the level of estrogen in the body. As a result, gonadotropin secretion increases due to blocking estrogen-negative feedback of LE, which stimulates the development of ovarian follicles (6).

Endometrial receptivity is critical for embryo implantation, and its impairment has been proven to be an important factor for infertility (7). In recent years, ultrasonic parameters, molecular markers in endometrial tissue and uterine secretions, endometrial microstructure, and hysteroscopy have been applied to evaluate endometrial receptivity (8). The preferred method for assessing endometrial receptivity is transvaginal ultrasound, and multiple ultrasonic indicators have been used to assess endometrial receptivity (9).

Numerous studies have reported on ovulation and pregnancy rates between LE and CC in PCOS women. However, relevant data on endometrial receptivity during an implantation window are limited, especially the non-invasive methods of assessment. Because LE and CC have different mechanisms for ovulation induction, we presumed that their endometrial receptivity during implantation windows is different, which affect subsequent pregnancy. The aim of this study was designed to compare the endometrial receptivity among an LE group, CC group, and natural cycle group, and to assess the predictive value for pregnancy of observed indicators.

\section{MATERIALS AND METHODS}

\section{Study Design}

We conducted a randomized controlled trial (RCT) study on PCOS patients with infertility in the outpatient clinic of obstetrics and gynecology and women's health care. All participants gave written informed consent on the basis of procedures granted by the Ethics Committee of The First Affiliated Hospital of Medical College of Xi'an Jiaotong University (XJTU1AF2019LSK-120). This study has been registered on China's clinical trials registration: www.chictr.org. cn (ChiCTR1900023423).

\section{Participants}

Two hundred and seventy-three PCOS patients were enrolled in the First Affiliated Hospital of Xi'an Jiaotong University from May 2018 to August 2019; among them three patients who declined to participate were excluded. All patients were between the ages of 22 and 38 years. The diagnostic criteria of PCOS was based on a modified Rotterdam criteria: menstrual abnormalities (oligomenorrhea, amenorrhea, or irregular uterine bleeding), combined with either hyperandrogenism or polycystic ovarian morphology (10). Hyperandrogenism was diagnosed according to either clinical manifestations or laboratory evidence. Clinical manifestations of hyperandrogenism included obesity or hirsutism. Obesity was defined as body mass index (BMI) $\geq 30.0 \mathrm{~kg} / \mathrm{m}^{2}$ (11). Hirsutism was defined as a FerrimanGallwey score of more than 6 in physical examination (12). Laboratory evidence of hyperandrogenemia was defined as total testosterone level exceeding the upper limit of normal levels on the basis of local laboratory criteria. Polycystic ovarian morphology was defined as the following criteria: the presence of at least 12 antral follicles measuring $2-9 \mathrm{~mm}$ in diameter in unilateral ovary or bilateral ovaries, and (or) an increased ovarian volume $(\geq 10 \mathrm{ml})$. Ovarian volume $=0.5 \times$ length diameter $\times$ transverse diameter $\times$ anteroposterior diameter (10). Patients were ruled out if they had congenital adrenal hyperplasia $(\mathrm{CAH})$, thyroid dysfunction, abnormal coagulation indicators, autoimmune disease or abnormal immune-related indicators, fallopian tube blocking, or a husband with abnormal semen. The baseline characteristics of all participants were recorded in detail. Serum sex hormone and anti-Mullerian 
hormone $(\mathrm{AMH})$ concentrations on 2-4 days of menses were tested.

\section{Intervention}

Patients were pretreated with lifestyle interventions, improved hyperandrogenism, hyperinsulinemia, and insulin resistance. Then 270 patients were randomly divided into three groups according to computer-generated random numbers, with 90 patients in each group. Patients in the LE group received LE for ovulation induction $(2.5 \mathrm{mg} /$ day on cycle days $5-9$ of menses for 1 cycle), and patients in the CC group received CC for ovulation induction (50 mg/day on cycle days 5-9 of menses for 1 cycle). Patients in the natural cycle group did not receive any drug for ovulation induction.

\section{Outcome Measures}

The follicle and endometrial thickness (ET) were detected using transvaginal ultrasound. Human chorionic gonadotropin (hCG) at a dose of 5000-10000 IU was used to trigger ovulation when a dominant follicle appeared (the average diameter $\geq 18 \mathrm{~mm}$ ). The indicators of endometrial receptivity of patients with successful ovulation in the three groups were tested 7 to 9 days after ovulation (defined as embryo implantation window period). Color Doppler was used to test resistance index (RI) and pulsatility index (PI) of the uterine artery. Additionally, 3-D power Doppler ultrasonography was used to calculate endometrial volume (EV), flow index (FI), vascularization index (VI), and vascularization flow index (VFI) (13). Meanwhile, uterine secretions were obtained using an embryo transfer catheter, which was linked to a syringe equipped with sodium chloride. The fluid was gently injected into the uterine cavity and then gently aspirated into the syringe (14). Integrin $\alpha v \beta 3$ and vascular endothelial growth factor (VEGF) concentrations in the uterine fluid were tested using an enzyme-linked immunosorbent assay.

The diagnostic criteria for pregnancy were as follows: (a) serum hCG level exceed $10 \mathrm{mIU} / \mathrm{mL}$ tested at 2 weeks after ovulation was considered to be biochemical pregnancy; (b) appearance of pregnant bursa or embryo in the uterine cavity was considered to be clinical pregnancy; and (c) appearance of a fetus with a heartbeat at 12 weeks of pregnancy was considered to be ongoing pregnancy (4).

The primary outcomes were endometrial receptivity and predictive value for pregnancy of observed parameters. The secondary outcomes were follicular development, ovulation, and pregnancy rates.

\section{Statistical Analysis}

Statistical analyses were performed using SPSS version 20.0. Normally distributed continuous data were given as mean \pm standard deviation, which were analyzed by the analysis of variance or Student's $t$-test. The enumeration variables were presented as number and percentage (\%), which were analyzed using chi-square test or Fisher's exact test. Endometrial receptivity of observed parameters was evaluated using receiver operating characteristic (ROC) curves. $P<0.05$ was considered statistically significant.

\section{RESULTS}

Two hundred and seventy-three PCOS patients were enrolled in this study, of which three patients were excluded. Hence, 270 patients were randomly assigned into three groups. There were 57,55 , and 11 patients with successful ovulation in the LE group, CC group, and natural cycle group, respectively. Endometrial

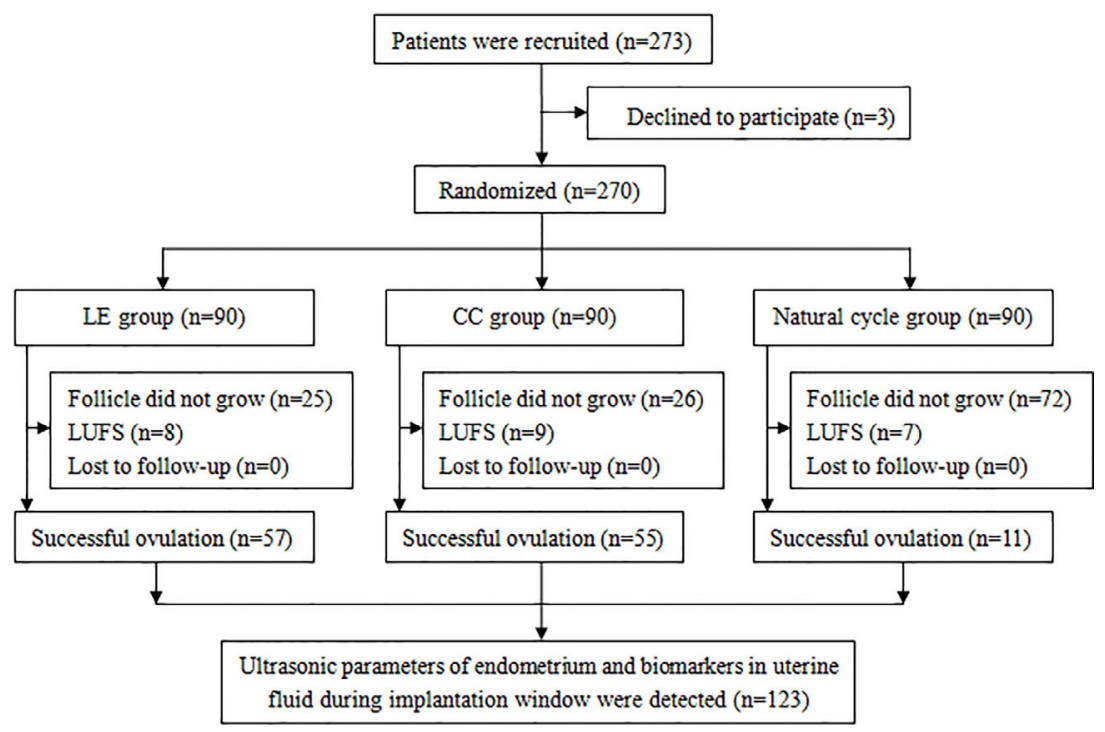

FIGURE 1 | Flow chart of the study. (LUFS, luteinized unruptured follicle syndrome). 
ultrasonic parameters, integrin $\alpha v \beta 3$, and VEGF in uterine fluid during the implantation window were tested (Figure 1).

\section{Basic Characteristic of Participants}

Table 1 indicates the basic data of patients among the three groups. The data show that no statistically significant differences were observed in the basic data of study subjects among the three groups $(P>0.05)$.

\section{Follicular Development and Ovulation Rate}

Table 2 reveals that the number of dominant follicles, number of ovulations, and successful ovulation rate in the LE group and CC group were significantly higher compared with the natural cycle group $(P<0.05)$, but the above indicators did not differ between the LE group and CC group $(P>0.05)$. Moreover, no statistically significant differences were observed in diameter of dominant follicle, dosage of hCG, and LUFS rate among the three groups $(P>0.05)$.

\section{Endometrial Receptivity}

Among patients with successful ovulation in the three groups (57 patients in the LE group, 55 patients in the CC group, and 11 patients in the natural cycle group), the parameters of endometrial receptivity during embryo implantation were tested. The data in Table 3 display that ET, EV, VI, FI, and VFI were significantly higher in the LE group, and integrin $\alpha v \beta 3$ and VEGF concentrations in uterine fluid were also significantly higher in the LE group than in the CC group and natural cycle group $(P<0.05)$, but no statistically significant differences were observed in the above parameters between the CC group and natural cycle group $(P>0.05)$. In addition, the data show that no statistically significant differences were observed in uterine PI and RI among the three groups $(P>0.05)$.

\section{Pregnancy Rates}

The data from Figure 2 present that biochemical pregnancy rate, clinical pregnancy rate, and ongoing pregnancy rate of the LE group and CC group were significantly higher compared with the natural cycle group $(28.9 \%$ or $21.1 \%$ vs. $6.7 \%, 25.6 \%$ or $13.3 \%$ vs. $4.4 \%, 23.3 \%$ or $11.1 \%$ vs.3.3\%) $(P<0.05)$. In addition, clinical pregnancy and ongoing pregnancy rates of the LE group were significantly higher than that of the CC group (25.6\% vs. $13.3 \%$, $23.3 \%$ vs. $11.1 \%)(P<0.05)$.

\section{Relationship Between Endometrial Receptivity Parameters and Pregnancy}

The patients with successful ovulation in this study were divided into four groups according to whether they were pregnant; 72 patients were in the no pregnancy group, 51 patients were in the biochemical pregnancy group, 39 patients were in the clinical

TABLE 1 | Basic data of study subjects among the three groups.

\begin{tabular}{|c|c|c|c|c|}
\hline Characteristics & LE group $(n=90)$ & CC group $(n=90)$ & Natural cycle group $(n=90)$ & $P$ value $^{\mathrm{a}}$ \\
\hline Age $\left(\right.$ years) ${ }^{b}$ & $28.5 \pm 7.6$ & $28.3 \pm 7.5$ & $29.1 \pm 6.1$ & 0.315 \\
\hline Weight $(\mathrm{kg})^{\mathrm{b}}$ & $63.2 \pm 8.7$ & $61.8 \pm 9.6$ & $61.5 \pm 10.4$ & 0.242 \\
\hline BMI $\left(\mathrm{kg} / \mathrm{m}^{2}\right)^{\mathrm{b}}$ & $24.9 \pm 8.4$ & $25.3 \pm 7.9$ & $24.7 \pm 6.6$ & 0.346 \\
\hline Waist circumference $(\mathrm{cm})^{\mathrm{b}}$ & $83.9 \pm 10.9$ & $81.6 \pm 11.3$ & $82.3 \pm 11.4$ & 0.138 \\
\hline Hip circumference $(\mathrm{cm})^{\mathrm{b}}$ & $95.1 \pm 10.1$ & $94.3 \pm 9.9$ & $96.4 \pm 10.2$ & 0.615 \\
\hline Waist-to-hip ratio ${ }^{b}$ & $0.9 \pm 0.4$ & $0.9 \pm 0.2$ & $0.8 \pm 0.3$ & 0.527 \\
\hline \multicolumn{5}{|l|}{ Basal concentration $^{\mathrm{b}}$} \\
\hline FSH (mlU/mL) & $7.3 \pm 1.6$ & $6.8 \pm 1.2$ & $7.2 \pm 1.9$ & 0.679 \\
\hline $\mathrm{LH}(\mathrm{mlU} / \mathrm{mL})$ & $10.2 \pm 2.1$ & $10.9 \pm 1.9$ & $11.3 \pm 2.3$ & 0.051 \\
\hline $\mathrm{PRL}(\mathrm{ng} / \mathrm{mL})$ & $14.2 \pm 4.0$ & $14.9 \pm 4.9$ & $13.6 \pm 5.8$ & 0.056 \\
\hline $\mathrm{P}(\mathrm{nmol} / \mathrm{L})$ & $1.6 \pm 0.8$ & $2.0 \pm 0.7$ & $1.9 \pm 0.9$ & 0.598 \\
\hline $\mathrm{E}_{2}(\mathrm{pmol} / \mathrm{L})$ & $59.1 \pm 10.2$ & $65.3 \pm 12.8$ & $61.9 \pm 12.5$ & 0.779 \\
\hline $\mathrm{T}(\mathrm{nmol} / \mathrm{L})$ & $1.5 \pm 0.7$ & $1.4 \pm 0.6$ & $1.3 \pm 0.8$ & 0.094 \\
\hline $\mathrm{AMH}(\mathrm{ng} / \mathrm{mL})^{\mathrm{b}}$ & $2.5 \pm 0.6$ & $2.7 \pm 0.8$ & $2.8 \pm 0.9$ & 0.213 \\
\hline Infertility duration (years) ${ }^{\mathrm{b}}$ & $2.4 \pm 0.7$ & $2.3 \pm 0.6$ & $2.0 \pm 0.5$ & 0.125 \\
\hline \multicolumn{5}{|l|}{ Family history ${ }^{c}$} \\
\hline Diabetes mellitus [n (\%)] & $7(7.8)$ & $4(4.4)$ & $6(6.7)$ & 0.644 \\
\hline Hypertension [n (\%)] & $9(10.0)$ & $8(8.9)$ & $10(11.1)$ & 0.884 \\
\hline
\end{tabular}

${ }^{a}$ Variance analysis, chi-square test or Fisher's exact test among the three groups. ${ }^{b}$ Data given as mean \pm SD. ${ }^{C}$ Data given as number (\%). FSH, follicle stimulating hormone; LH, luteinizing hormone; PRL, prolactin; P, progesterone; $E_{2}$, estradiol; $T$, testosterone.

TABLE 2 | Comparison of follicular development and ovulation rate among the three groups.

\begin{tabular}{|c|c|c|c|c|}
\hline Parameters & LE group $(n=90)$ & CC group $(n=90)$ & Natural cycle group $(n=90)$ & $P$ value $^{\mathrm{a}}$ \\
\hline Number of dominant follicle ${ }^{b}$ & $1.4 \pm 0.5^{\star}$ & $1.5 \pm 0.6^{\star}$ & $0.2 \pm 0.1$ & 0.022 \\
\hline Diameter of dominant follicle $(\mathrm{mm})^{\mathrm{b}}$ & $20.3 \pm 6.1$ & $20.4 \pm 5.9$ & $19.6 \pm 5.8$ & 0.897 \\
\hline Number of ovulation ${ }^{\mathrm{b}}$ & $1.2 \pm 0.5^{\star}$ & $1.1 \pm 0.4^{\star}$ & $0.2 \pm 0.1$ & 0.025 \\
\hline Dosage of hCG $(I U)^{b}$ & $8164.3 \pm 128.5$ & $8456.7 \pm 139.1$ & $9539.4 \pm 124.6$ & 0.052 \\
\hline Successful ovulation [n (\%)] ${ }^{\mathrm{c}}$ & $57(63.3)^{\star}$ & $55(61.1)^{\star}$ & $11(12.2)$ & 0.000 \\
\hline LUFS $[n(\%)]^{\mathrm{C}}$ & $8(8.9)$ & $9(10.0)$ & $7(7.8)$ & 0.872 \\
\hline
\end{tabular}

${ }^{a}$ Variance analysis or chi-square test among the three groups. ${ }^{b}$ Data given as mean $\pm S D$. ${ }^{c}$ Data given as number (\%). Vs. natural cycle group, ${ }^{\star} P<0.05$. 
TABLE 3 | Comparison of endometrial receptivity among the three groups.

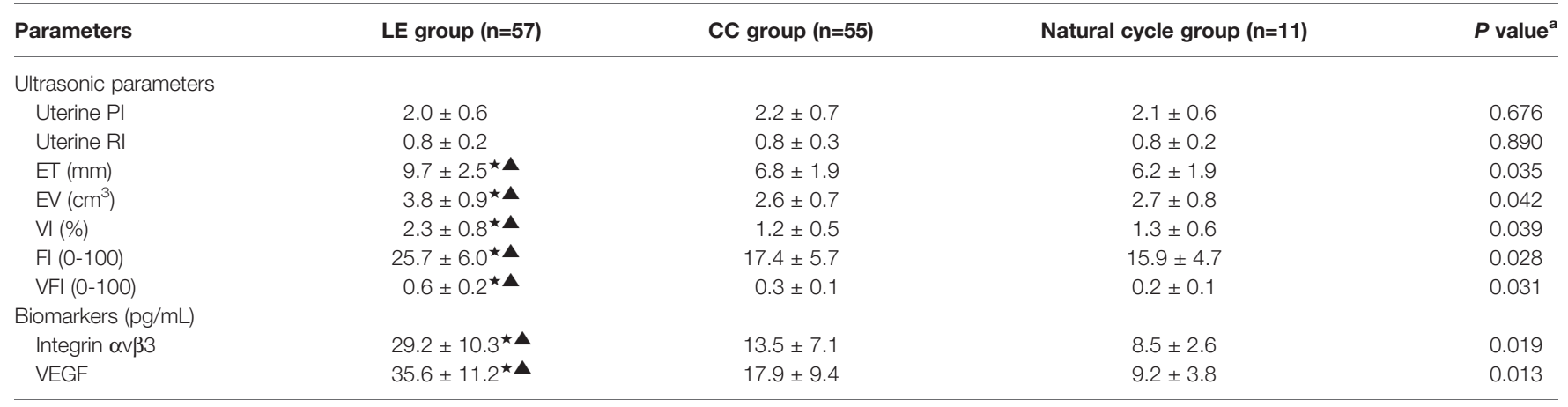

Data given as mean $\pm S D$. ${ }^{a}$ Variance analysis among the three groups. Vs. natural cycle group, ${ }^{\star} P<0.05$. Vs. CC group, ${ }^{\mathbf{\Delta}} P<0.05$.

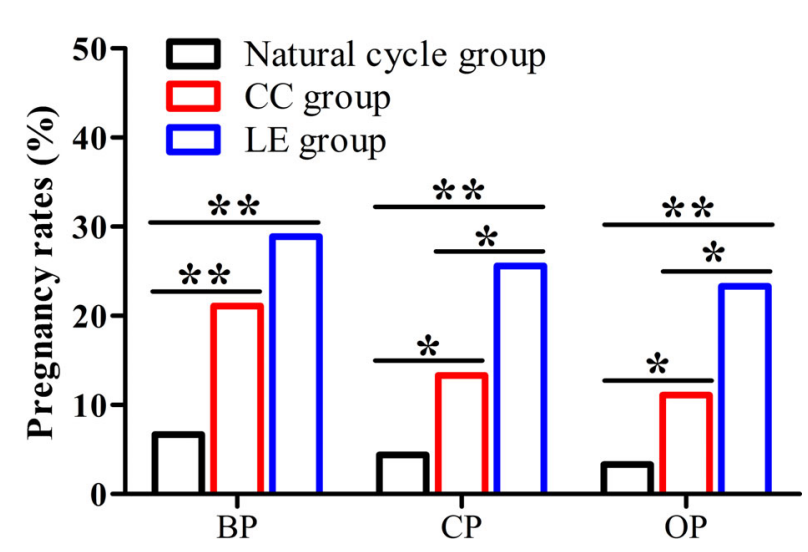

FIGURE 2 | Pregnancy rates among the three groups. (BP, biochemical pregnancy; $\mathrm{CP}$, clinical pregnancy; $\mathrm{OP}$, ongoing pregnancy), ${ }^{\star} P<0.05$, ${ }^{\star \star} P<0.01$.

pregnancy group, and 34 patients were in the ongoing pregnancy group. The data from Table 4 suggest that endometrial VI, FI and VFI, integrin $\alpha v \beta 3$, and VEGF concentrations in the uterine fluid of all pregnancy groups were significantly higher compared with the no pregnancy group $(P<0.05)$. Moreover, the above parameters in ongoing pregnancy were significantly higher than that of the biochemical pregnancy group $(P<0.05)$. However, no statistically significant differences were observed in uterine PI and RI, ET, and EV among the four groups $(P>0.05)$.

\section{Predictive Value for Pregnancy During Implantation Window}

Figure 3 demonstrates the predictive value of various parameters during the implantation window for pregnancy in PCOS patients. The best ultrasonic parameter for predicting pregnancy was endometrial FI (AUC=0.889); the cut-off of 22.9 provided a sensitivity of $92.7 \%$ and a specificity of $62.6 \%$. The data show that integrin $\alpha v \beta 3$ in uterine fluid during the implantation window had better predictive value (AUC $=0.876$ ) compared with VEGF; the cut-off of $29.6 \mathrm{pg} / \mathrm{mL}$ provided a sensitivity of $92.4 \%$ and a specificity of $54.8 \%$.

\section{DISCUSSION}

Ovulation induction is regarded as an important therapeutic method for PCOS women with infertility. Previous studies reported that the ovulation rate of CC in PCOS women was $70 \%-80 \%$. Nevertheless, the pregnancy rate was relatively low (25\%-60\%) (2). In addition to the side effects on cervical mucus (15), the main reason is that CC impaired endometrial development, which resulted in endometrial thinning and lower receptivity in PCOS women (16). Furthermore, approximately $15 \%-40 \%$ of women with PCOS are resistant to CC for ovulation induction (17). Because LE does not affect the central feedback mechanisms, they remain intact, making it superior to CC in ovulation induction. Hence, LE is recommended as first-line medication for ovulation induction in PCOS women according to the evidence-based medical evidence guidelines released internationally in 2018 (18).

Many studies were conducted to compare ovulation rates between CC and LE in women with PCOS. Literature has reported that LE was associated with higher cumulative ovulation rates among infertile patients with PCOS compared with CC (19). A recent meta-analysis of RCTs showed that LE significantly increased ovulation rates in women with PCOS (20). However, data in our study showed that the number of dominant follicles, number of ovulations, and successful ovulation rates in the LE group and CC group were significantly higher compared with the natural cycle group, but the above indicators did not differ between the LE group and CC group. The diverse outcomes perhaps related to the study design, different cycles, and dosages of LE and CC, race, and pre-treatment.

The endometrium has only a very short period of maximum sensitivity for embryo implantation in the normal menstrual cycle, about 7 to 9 days after ovulation. This period is defined as the window of implantation, and is the most appropriate time for embryo implantation (21). Infertility in PCOS women is related to anovulation and endometrial dysfunction. Both appropriate ET and adequate endometrial blood perfusion are crucial to embryo implantation. Therefore, the angiogenesis is active and the endometrial blood flow is increased in the early stage of embryo implantation, which offers support for pregnancy. With advances in diagnostic ultrasonography, several ultrasonographic indicators have been used to estimate endometrial receptivity, including 
TABLE 4 | Comparison of endometrial receptivity parameters among the four groups.

\begin{tabular}{|c|c|c|c|c|c|}
\hline Parameters & $\begin{array}{l}\text { No pregnancy group } \\
\qquad(\mathrm{n}=72)\end{array}$ & $\begin{array}{l}\text { Biochemical pregnancy group } \\
\qquad(\mathrm{n}=51)\end{array}$ & $\begin{array}{l}\text { Clinical pregnancy group } \\
\qquad(\mathrm{n}=39)\end{array}$ & $\begin{array}{l}\text { Ongoing pregnancy group } \\
\qquad(n=34)\end{array}$ & $\begin{array}{c}P \\
\text { value }^{\mathrm{a}}\end{array}$ \\
\hline \multicolumn{6}{|c|}{ Ultrasonic parameters } \\
\hline Uterine RI & $0.8 \pm 0.3$ & $0.8 \pm 0.3$ & $0.8 \pm 0.3$ & $0.8 \pm 0.2$ & 0.324 \\
\hline ET (mm) & $9.6 \pm 2.3$ & $9.9 \pm 2.9$ & $10.2 \pm 3.0$ & $9.9 \pm 2.8$ & 0.225 \\
\hline $\mathrm{EV}\left(\mathrm{cm}^{3}\right)$ & $2.8 \pm 0.8$ & $2.9 \pm 0.9$ & $2.9 \pm 0.8$ & $3.0 \pm 0.9$ & 0.210 \\
\hline VI (\%) & $1.5 \pm 0.6$ & $2.2 \pm 0.8^{\star}$ & $2.5 \pm 0.8^{\star}$ & $2.9 \pm 0.9 \star \boldsymbol{\Lambda}$ & 0.044 \\
\hline \multicolumn{6}{|c|}{ Biomarkers (pg/mL) } \\
\hline Integrin $\alpha v \beta 3$ & $11.2 \pm 3.9$ & $26.8 \pm 7.3^{\star}$ & $32.6 \pm 7.4^{\star}$ & $40.3 \pm 10.2^{\star \boldsymbol{\Delta}}$ & 0.015 \\
\hline VEGF & $16.7 \pm 5.1$ & $29.5 \pm 9.4^{\star}$ & $41.5 \pm 10.9^{\star}$ & $50.2 \pm 11.5^{\star \boldsymbol{\Delta}}$ & 0.020 \\
\hline
\end{tabular}

Data given as mean $\pm S D$. ${ }^{a}$ Variance analysis among the three groups. Vs. no pregnancy group, ${ }^{\star} P<0.05$. Vs. biochemical pregnancy group, ${ }^{\mathbf{\Delta}} P<0.05$.

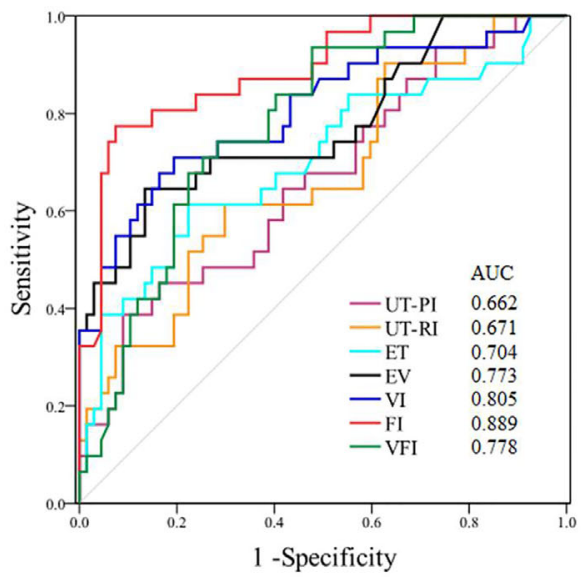

B

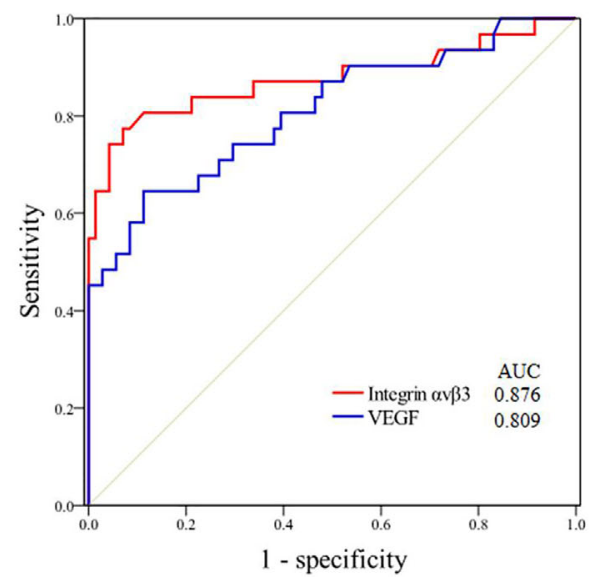

FIGURE 3 | ROC curves of the predictive value for pregnancy during the implantation window in patients with PCOS. (UT-PI, PI of uterine artery; UT-RI, RI of uterine artery RI). (A) Ultrasonic parameters. (B) Biomarkers.

uterine arterial blood flow, endometrial blood flow, and vascularisation parameters (22).

At present, 3-D energy Doppler ultrasonic scanning has been applied to estimate endometrial receptivity (23). Scholars proved that this technology has better predictive value for endometrial receptivity than 2-D ultrasound, which has been used for in vitro fertilization-embryo transfer (IVF-ET) (24). Yaman et al. reported that 3-D energy Doppler offers a unique tool to reflect the vascular structure of the whole endometrium and the supply of blood flow, which could be used as a predictor of pregnancy in patients who accepted assisted reproductive technology (25). Our findings indicate that patients in the LE group had significantly higher ET, EV, VI, FI, and VFI than patients in the CC group. Selim et al. found that ET was significantly thicker in the LE cycles compared to CC cycles (26), which were similar to our results. Roy et al. pointed out that the effect of LE displayed a better endometrial response and pregnancy rate than CC (27). Similarly, Al-Obaidi et al. reported that the application of LE in PCOS patients had a better curative effect on endometrial receptivity compared with CC (28).
Additionally, the data in our study revealed that the endometrial VI, FI, and VFI of all pregnancy groups were significantly higher than that of the no pregnancy group, and the above indicators in ongoing pregnancy were significantly higher than those of the biochemical pregnancy group. In conclusion, the data in our study revealed that the endometrial receptivity during the implantation window in LE is superior to $\mathrm{CC}$ for ovulation induction among PCOS women.

Uterine fluid includes a variety of cytokines, which provide a suitable microenvironment for embryo implantation (29). Previous studies confirmed that intrauterine fluid aspiration during the window of implantation did not affect the success rate of pregnancy in women seeking pregnancy, such as patients who accepted intrauterine insemination or IVF-ET $(30,31)$. In addition, intrauterine fluid aspiration also had no effect on pregnancy in infertile patients with endometriosis, or patients with idiopathic infertility or luteal phase deficiency (32). Similar to previous studies, the safety of this method has been proven in our previous studies, which selected fertile women and patients with unexplained infertility as subjects (33). Data in our study revealed that integrin 
$\alpha v \beta 3$ and VEGF concentrations in uterine fluid were significantly higher in the LE group compared with the CC group and natural cycle group. Integrin $\alpha v \beta 3$ and VEGF concentrations in the uterine fluid of all pregnancy groups were significantly higher compared with the no pregnancy group. Additionally, integrin $\alpha v \beta 3$ and VEGF concentrations in ongoing pregnancy were significantly higher compared with the biochemical pregnancy group. The concentrations of biochemical indicators in endometrial tissue and uterine fluid are related to embryo implantation. Boomsma et al. revealed that cytokines secreted by the endometrium provide a non-invasive method for evaluating endometrial receptivity and predicting embryo implantation (34).

According to the results of this study, clinical pregnancy and ongoing pregnancy rates of the LE group were significantly higher than in the CC group, which was consistent with previous studies. Wang et al. pointed out that LE improves clinical pregnancy and live birth rate compared to CC in women with PCOS (35). In another study, Roque et al. compared the results of CC versus LE for ovulation induction in PCOS women, and reported that LE was superior in pregnancy rates and live birth rates (36). Similarly, a recent study by $\mathrm{Hu}$ et al. found that LE significantly increased pregnancy rates and live birth rates in women with PCOS (20). Differences in pregnancy rates between patients with PCOS taking LE or CC may be explained by different endometrial receptivity during the implantation window, in addition to the changes of cervical mucus. Furthermore, our research also assessed the predictive value for pregnancy of observed indicators; data displayed that endometrial FI during the implantation window had the highest predictive value for pregnancy in patients with PCOS, the cut-off of 22.9 provided a sensitivity of $92.7 \%$ and a specificity of $62.6 \%$. Integrin $\alpha v \beta 3$ in uterine fluid during the implantation window had better predictive value than VEGF; the cut-off of $29.6 \mathrm{pg} / \mathrm{mL}$ provided a sensitivity of $92.4 \%$ and a specificity of $54.8 \%$.

The present study still has some limitations. First, the RCT study was done in a single center. Additionally, the evaluative parameters were limited to one ovulation cycle, and live birth rate was not followed up. Second, endometrial receptivity is associated with many factors, including insulin resistance, hyperinsulinemia, thyroid hormone levels, abnormal immunerelated indicators, haematologic parameters, and other cytokines. Therefore, multi-center studies with large sample sizes should be designed to verify the results of this research in the future.

\section{REFERENCES}

1. March WA, Moore VM, Willson KJ, Phillips DI, Norman RJ, Davies MJ. The prevalence of polycystic ovary syndrome in a community sample assessed under contrasting diagnostic criteria. Hum Reprod (2010) 25:544-51. doi: 10.1093/humrep/dep399

2. Wallace KL, Johnson V, Sopelak V, Hines R. Clomiphene citrate versus letrozole: molecular analysis of the endometrium in women with polycystic ovary syndrome. Fertil Steril (2011) 96:1051-6. doi: 10.1016/j.fertnstert.2011.07.1092

3. Lim SS, Davies MJ, Norman RJ, Moran LJ. Overweight, obesity and central obesity in women with polycystic ovary syndrome: a systematic review and meta-analysis. Hum Reprod Update (2012) 18:618-37. doi: 10.1093/humupd/dms030

\section{CONCLUSION}

Endometrial receptivity during the implantation window of LE for ovulation induction is superior to CC in PCOS women, which may be associated with higher clinical pregnancy and ongoing pregnancy rates. Endometrial FI and integrin $\alpha v \beta 3$ in uterine secretion during the implantation window could be preferable non-invasive predictor markers for pregnancy.

\section{DATA AVAILABILITY STATEMENT}

All datasets generated for this study are included in the article/ supplementary material.

\section{ETHICS STATEMENT}

The studies involving human participants were reviewed and approved by First Affiliated Hospital of Xi'an Jiaotong University Institutional Review Board. The patients/participants provided their written informed consent to participate in this study. Written informed consent was obtained from the individual(s) for the publication of any potentially identifiable images or data included in this article.

\section{AUTHOR CONTRIBUTIONS}

LW and XY contributed to the conception and design of the study. SL provided participants. FL and EB participated in data collection and patients' follow-up. All authors contributed to the article and approved the submitted version.

\section{FUNDING}

This study was supported by the Natural Science Basic Research Program of Shaanxi (Program No.2019JM-569) and by the Institutional Foundation of the First Affiliated Hospital of Xi'an Jiaotong University (Program No.2019ZYTS-03).
4. Wang L, Wen X, Lv S, Zhao J, Yang T, Yang X. Comparison of endometrial receptivity of clomiphene citrate versus letrozole in women with polycystic ovary syndrome: a randomized controlled study. Gynecol Endocrinol (2019) 35:862-5. doi: 10.1080/09513590.2019.1612358

5. Gadalla MA, Huang S, Wang R, Norman RJ, Abdullah SA, El Saman AM, et al. Effect of clomiphene citrate on endometrial thickness, ovulation, pregnancy and live birth in anovulatory women: systematic review and meta-analysis. Ultrasound Obstet Gynecol (2018) 51:64-76. doi: 10.1002/ uog. 18933

6. Franik S, Eltrop SM, Kremer JA, Kiesel L, Farquhar C. Aromatase inhibitors (letrozole) for subfertile women with polycystic ovary syndrome. Cochrane Database Syst Rev (2018) 5:CD010287. doi: 10.1002/14651858.CD010287.pub3 
7. Lessey BA, Young SL. What exactly is endometrial receptivity? Fertil Steril (2019) 111:611-7. doi: 10.1016/j.fertnstert.2019.02.009

8. Craciunas L, Gallos I, Chu J, Bourne T, Quenby S, Brosens JJ, et al. Conventional and modern markers of endometrial receptivity: a systematic review and meta-analysis. Hum Reprod Update (2019) 25:202-23. doi: 10.1093/humupd/dmy044

9. Liu H, Zhang J, Wang B, Kuang Y. Effect of endometrial thickness on ectopic pregnancy in frozen embryo transfer cycles: an analysis including 17244 pregnancy cycles. Fertil Steril (2019) 19:32316-7. doi: 10.1016/j.fertnstert. 2019.09.003

10. Endocrinology Group and Guideline Expert Group of Chinese Medical Association Obstetrics and Gynecology Branch. Chinese guidelines for the diagnosis and treatment of polycystic ovary syndrome. Chin J Obstet Gynecol (2018) 53:2-6. doi: 10.3760/cma.j.issn.0529-567x.2018.01.002

11. Teede HJ, Misso ML, Costello MF, Dokras A, Joop L, Moran L, et al. Recommendations from the international evidence-based guideline for the assessment and management of polycystic ovary syndrome. Fertil Steril (2018) 110:364-79. doi: 10.1016/j.fertnstert.2018.05.004

12. Chen ZJ, Shi Y, Sun Y, Zhang B, Liang X, Cao Y, et al. Fresh versus frozen embryos for infertility in the polycystic ovary syndrome. N Engl J Med (2016) 375:523-33. doi: 10.1056/NEJMoa1513873

13. Kupesic S, Bekavac I, Bjelos D, Kurjak A. Assessment of endometrial receptivity by transvaginal color Doppler and three-dimensional power Doppler ultrasonography in patients undergoing in vitro fertilization procedures. J Ultrasound Med (2001) 20:125-34. doi: 10.7863/jum.2001.20.2.125

14. Rahiminejad ME, Moaddab A, Ebrahimi M, Rabiee S, Zamani A, Ezzati M, et al. The relationship between some endometrial secretion cytokines and in vitro fertilization. Iran J Reprod Med (2015) 13:557-62. doi: 10.1007/s00192007-0485-7

15. Al-Fozan H, Al-Khadouri M, Tan SL, Tulandi T. A randomized trial of letrozole versus clomiphene citrate in women undergoing superovulation. Fertil Steril (2004) 82:1561-3. doi: 10.1016/j.fertnstert.2004.04.070

16. Bao SH, Sheng SL, Peng YF, Lin QD. Effects of letrozole and clomiphene citrate on the expression of HOXA10 and integrin alpha v beta 3 in uterine epithelium of rats. Fertil Steril (2009) 91:244-8. doi: 10.1016/j.fertnstert.2007.11.024

17. Wang L, Qi H, Baker PN, Zhen Q, Zeng Q, Shi R, et al. Altered circulating inflammatory cytokines are associated with anovulatory polycystic ovary syndrome (PCOS) women resistant to clomiphene citrate treatment. Med Sci Monit (2017) 23:1083-9. doi: 10.12659/msm.901194

18. Costello MF, Misso ML, Balen A, Boyle J, Devoto L, Garad RM, et al. Evidence summaries and recommendations from the international evidence-based guideline for the assessment and management of polycystic ovary syndrome: assessment and treatment of infertility. Hum Reprod Open (2019) 2019:hoy021. doi: 10.1093/hropen/hoy021. eCollection 2019.

19. Legro RS, Brzyski RG, Diamond MP, Coutifaris C, Schlaff WD, Casson P, et al. Letrozole versus clomiphene for infertility in the polycystic ovary syndrome. N Engl J Med (2014) 371:119-29. doi: 10.1056/NEJMoa1313517

20. Hu S, Yu Q, Wang Y, Wang M, Xia W, Zhu C. Letrozole versus clomiphene citrate in polycystic ovary syndrome: a meta-analysis of randomized controlled trials. Arch Gynecol Obstet (2018) 297:1081-8. doi: 10.1007/ s00404-018-4688-6

21. Paulson RJ. Introduction: endometrial receptivity: evaluation, induction and inhibition. Fertil Steril (2019) 111:609-10. doi: 10.1016/j.fertnstert.2019.02.029

22. Kim A, Jung H, Choi WJ, Hong SN, Kim HY. Detection of endometrial and subendometrial vasculature on the day of embryo transfer and prediction of pregnancy during fresh in vitro fertilization cycles. Taiwan J Obstet Gynecol (2014) 53:360-5. doi: 10.1016/j.tjog.2013.05.007

23. Mohsen IA, Elkattan E, Nabil H, Khattab S. Effect of Metformin treatment on endometrial vascular indices in anovulatory obese/overweight women with polycystic ovarian syndrome using three-dimensional power Doppler ultrasonography. J Clinic Ultrasound (2013) 41:275-82. doi: 10.1002/ jcu.22006
24. Schild RL, Holthaus S, Dalquen J, Fimmers R, Dorn C, Van Der Ven H, et al. Quantitative assessment of subendometrial blood flow by three-dimensionalultrasound is an important predictive factor of implantation in an in-vitro fertilization programme. Hum Reprod (2000) 15:89-94. doi: 10.1093/humrep/ 15.1.89

25. Yaman C, Mayer R. Three-dimensional ultrasound as a predictor of pregnancy in patients undergoing ART. J Turk Ger Gynecol Assoc (2012) 13:128-34. doi: 10.5152/jtgga.2012.15. eCollection 2012.

26. Selim MF, Borg TF. Letrozole and clomiphene citrate effect on endometrial and subendometrial vascularity in treating infertility in women with polycystic ovary syndrome. J Gynecol Surg (2012) 28:405-10. doi: 10.1089/ gyn.2012.0033

27. Roy KK, Baruah J, Singla S, Sharma JB, Singh N, Jain SK, et al. A prospective randomized trial comparing the efficacy of Letrozole and Clomiphene citrate in induction of ovulation in polycystic ovarian syndrome. J Hum Reprod Sci (2012) 5:20-5. doi: 10.4103/0974-1208.97789

28. Al-Obaidi MT, Ali ZH, Al-Saadi WI, Al-Wasiti EAR, Al-Aubaidy H. Impact of letrozole versus clomiphene citrate on endometrial receptivity in Iraqi women with polycystic ovarian syndrome. J Clin Pharm Ther (2019) 44:61822. doi: $10.1111 /$ jcpt.12831

29. Edgell TA, Evans J, Lazzaro L, Boyes K, Sridhar M, Catt S, et al. Assessment of potential biomarkers of pre-receptive and receptive endometrium in uterine fluid and a functional evaluation of the potential role of CSF3 in fertility. Cytokine (2018) 111:222-9. doi: 10.1016/j.cyto.2018.08.026

30. Florio P, Bruni L, De Falco C, Filardi G, Torricelli M, Reis FM, et al. Evaluation of endometrial urocortin secretion for prediction of pregnancy after intrauterine insemination. Clin Chem (2008) 54:350-5. doi: 10.1373/ clinchem.2007.094987

31. Van der Gaast MH, Beier-Hellwig K, Fauser BC, Beier HM, Macklon NS. Endometrial secretion aspiration prior to embryo transfer does not reduce implantation rates. Reprod BioMed Online (2003) 7:105-9. doi: 10.1016/ s1472-6483(10)61737-3

32. Mikolajczyk M, Wirstlein P, Skrzypczak J. The impact of leukemia inhibitory factor in uterine flushing on the reproductive potential of infertile women: a prospective study. Am J Reprod Immunol (2007) 58:65-74. doi: 10.1111/ j.1600-0897.2007.00492.x

33. Wang L, Lv SL, Mao WJ, Pei ML, Yang XF. Assessment of endometrial receptivity during implantation window in women with unexplained infertility. Gynecol Endocrinol (2020) 36:917-21. doi: 10.1080/09513590. 2020.1727433

34. Boomsma CM, Kavelaars A, Eijkemans MJ, Lentjes EG, Fauser BC, Heijnen CJ, et al. Endometrial secretion analysis identifies a cytokine profile predictive of pregnancy in IVF. Hum Reprod (2009) 24:1427-35. doi: 10.1093/humrep/dep011

35. Wang R, Li W, Bordewijk EM, Legro RS, Zhang H, Wu X, et al. First-line ovulation induction for polycystic ovary syndrome: an individual participant data meta-analysis. Hum Reprod Update (2019) 25:717-32. doi: 10.1093/ humupd/dmz029

36. Roque M, Tostes AC, Valle M, Sampaio M, Geber S. Letrozole versus clomiphene citrate in polycystic ovary syndrome: systematic review and meta-analysis. Gynecol Endocrinol (2015) 31:917-21. doi: 10.3109/ 09513590.2015.1096337

Conflict of Interest: The authors declare that the research was conducted in the absence of any commercial or financial relationships that could be construed as a potential conflict of interest.

Copyright (c) 2021 Wang, Lv, Li, Bai and Yang. This is an open-access article distributed under the terms of the Creative Commons Attribution License (CC BY). The use, distribution or reproduction in other forums is permitted, provided the original author(s) and the copyright owner(s) are credited and that the original publication in this journal is cited, in accordance with accepted academic practice. No use, distribution or reproduction is permitted which does not comply with these terms. 